\title{
Large-area bi-component processing of organic semiconductors by spray deposition and spin coating with orthogonal solvents
}

\author{
Emanuele Treossi • Andrea Liscio • Xinliang Feng • \\ Vincenzo Palermo • Klaus Müllen • Paolo Samorì
}

Received: 2 August 2008 / Accepted: 24 November 2008 / Published online: 7 February 2009

(c) Springer-Verlag 2009

\begin{abstract}
Micrometre-thick uniform layers of a polymeric semiconductor (poly(3-hexylthiophene), P3HT) have been fabricated from solution by spray deposition making use of a commercial airbrush. Multi-scale characterization by optical microscopy and atomic force microscopy revealed the formation of smooth layers featuring reproducible patterns of spatially correlated micron-sized holes. This morphology was found to be uniform over the whole sample surface, on millimetre scale. On this micro-patterned P3HT layer an orthogonal solvent (i.e. a solvent which does not
\end{abstract}

This work was supported by the ESF-SONS2-SUPRAMATES project, the German Science Foundation (Mu 334/28-1), the Regione Emilia-Romagna PRIITT Nanofaber Net-Lab as well as the EU through the projects Marie Curie EST-SUPER (MEST-CT-2004-008128) and the RTN PRAIRIES (MRTN-CT-2006-035810).

E. Treossi - A. Liscio · V. Palermo

Istituto per la Sintesi Organica e la Fotoreattività, Consiglio

Nazionale delle Ricerche, via Gobetti 101, 40129 Bologna, Italy

V. Palermo

e-mail: palermo@isof.cnr.it

X. Feng $\cdot$ K. Müllen

Max-Planck Institute for Polymer Research, Ackermannweg 10, 55124 Mainz, Germany

K. Müllen

e-mail: muellen@mpip-mainz.mpg.de

P. Samorì $(\bowtie)$

Nanochemistry Laboratory, ISIS-CNRS 7006, Université Louis Pasteur, 8 allée Gaspard Monge, 67000 Strasbourg, France e-mail: samori@isis-ulp.org

P. Samorì

Istituto per la Sintesi Organica e la Fotoreattività, Consiglio Nazionale delle Ricerche, via Gobetti 101, 40129 Bologna, Italy dissolve the P3HT) has been employed to deposit either by spin coating or by drop casting a second organic semiconductor. While spin-coated films exhibited nano-crystals of an alkylated perylene tetracarboxy diimide (PDI) preferentially grown into the micro-fabricated holes, drop-cast films displayed crystalline PDI fibres adsorbed on the patterned surface in random positions.

PACS 68.37.Ps $\cdot$ 68.55.Am $\cdot$ 68.55.Ag $\cdot$ 81.15.Rs . 81.16.Dn

\section{Introduction}

The deposition of organic functional materials based on small molecules or polymers [1] on solid surfaces is of paramount importance for applications in various scientific and technological fields ranging from organic electronics to paints and protective coatings [2-4].

The simplest, cheapest and industrially more relevant method of deposition is the processing from solution. Although being very simple, the deposition of a solution on a surface, due to the concomitant solvent evaporation and formation of a solid layer of material, is a physically complex process which strongly depends on the components used and phases formed (solute, solvent, substrate, atmosphere) as well as on the processing method employed (dip coating, spin coating, drop casting, etc.) $[5,6]$.

In the field of organic electronics [7-10], the situation is even more complex, because the typically used organic semiconductors often show complex self-assembly behaviours [11]. In many cases, solubility of large organic molecules is quite low, and this limits the type and amount of 
material which can be deposited from solution, e.g. by simple spin coating. For all these reasons, the possibility to deposit large amounts of organic material by an alternative and unconventional method, such as spray deposition, has been extensively studied by several groups. In spray deposition, a continuous flow of carrier gas (air, nitrogen or argon) is used to disperse the solution into microscopic droplets, which collides with the surface and sub-sequentially evaporate. The amount of deposited material is directly proportional to the deposition (i.e. exposure) time allowing the formation of very thick and uniform layers.

Spray deposition of organic semiconductors has been performed both in air, using commercial airbrushes [12, 13], or in vacuum [14-16], using shadow masks to fabricate micro-pixels [17] or computer-controlled micro-nozzles to create more complex patterns and electrodes [18]. Because of the elevated film thickness and the peculiar deposition conditions such as the high quantity of materials adsorbed at surfaces per unit time, the final film morphology is poorly influenced by the substrate nature [14]. Solar cells [12, 13, 16, 19] and LEDs [14] have been fabricated starting from spray-deposited organic layers, and the possibility to obtain complex layer-by-layer growth of different materials has been demonstrated [20]. Laser techniques have been successfully used to study the dynamics of the process [21]. Overall, the morphology of spray-deposited samples depends on a variety of experimental parameters, most of which are difficult to be controlled and modelled [20].

Herein we study the spray deposition of a common organic semiconductor, poly(3-hexylthiophene) (P3HT), a molecular system which has been successfully used for the fabrication of solar cells and field-effect transistors [22-24]. The spray-deposited P3HT films have then been employed as a macroscopic template to direct the self-assembly of a second organic semiconductor, such as an alkyl substituted perylene-bis(dicarboximide) (PDI) [25-30], deposited (i.e. via either spin coating or drop casting) using an orthogonal solvent. The obtained films have been characterized across multiple length scales by optical microscopy as well as atomic force microscopy.

\section{Experimental}

P3HT was purchased from BASF $\left(M_{w}=20 \mathrm{KDa}\right)$ while $N, N^{\prime}$-bis(1-ethylpropyl)-3,4:9,10-perylenebis(dicarboximide) (PDI) was synthesized as previously described [31].

A $1.5 \mathrm{~g} / \mathrm{L}$ solution of P3HT in chloroform has been sprayed by air brush on native $\mathrm{SiO}_{x}$ layer grown on $\mathrm{Si}$ and glass substrates by systematically changing the deposition time. Silicon (100) and glass substrates have been cleaned using a standard RCA procedure [32].

A commercial air brush (Paasche V) with a nozzle diameter of $0.1 \mathrm{~mm}$ has been used with pressurized argon
(3.5 atm) as carrier gas. PDI was deposited by spin coating $(1600 \mathrm{rpm})$ a saturated methanol $(\mathrm{MeOH})$ solution. All solvents employed were HPLC grade.

Atomic force microscopy (AFM) [33-35] was performed with an Autoprobe CP from Veeco, recording both the height signal (output of the feedback signal) and the phase signal (phase lag of the tip oscillation respect to the piezo oscillation) which is extremely sensitive to structural heterogeneities on the sample surface [36-38].

The surface periodicity has been studied with fast Fourier transform (FFT) as well as correlation analyses. In the correlation technique, two images are compared after being translated of $x, y$ pixels, in respect to each other, to detect the presence of periodic structures/features in the image. While FFT singles out the dominant periodicity present on the surface, correlation analysis does not filter specific frequencies but preserve all frequencies that are represented in the repeated structure [39].

The analyzed image can be defined as a matrix of $M \times N$ pixels, each pixel having a value $I(x, y)$ (which in AFM corresponds to topographic height and in OM to pixel luminosity).

The 2D correlation function $S(x, y)$ is a matrix of $M \times N$ pixels, which is calculated in each point as:

$S(x, y)=\sum_{k=0}^{M-1-x} \sum_{l=0}^{N-1-y} I(k, l) \times I(k+x, l+y)$.

$S(x, y)$ is proportional to the probability of finding two similar objects translated of $x, y$ pixels, in respect to each other on the substrate plane, similarly to the pair correlation function of inter-atomic distances in solid materials.

The surface flatness was characterized by its root mean square roughness $\left(R_{\mathrm{RMS}}\right)$.

$R_{\mathrm{RMS}}=\sqrt{\frac{1}{M N} \sum_{k}^{M-1} \sum_{l}^{N-1}\left[z\left(x_{k}, y_{l}\right)-\bar{z}\right]^{2}}$

with $z\left(x_{k}, y_{l}\right)$ being the height value of the pixel in location $\left(x_{k}, y_{l}\right)$ and $\bar{z}$ being the mean height of the pixel calculated from the $M \times N$ values.

\section{Results and discussion}

Nozzle-sample distance and argon pressure have been optimized, after systematic tests, to the values of $3 \mathrm{~cm}$ and $3.5 \mathrm{~atm}$, respectively. At larger separation distances the sprayed area increases, although poorly uniform films are obtained and deposition speeds are low [20]. At shorter distances deposition speed is greater than solvent evaporation rate leading to solution accumulation and drainage on the substrate surface. 
Fig. 1 Optical microscopy images of spray-deposited P3HT films at different deposition time. White arrows indicate small holes due to droplet impact
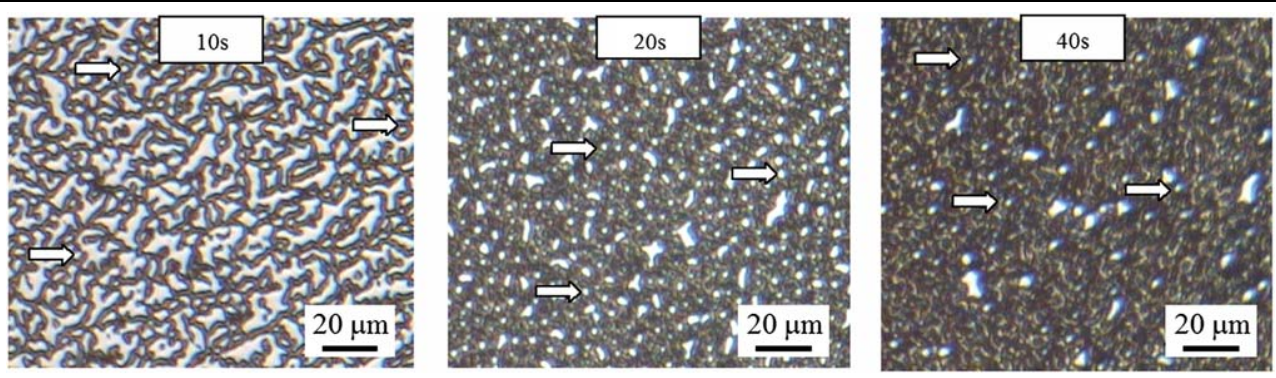

Fig. 2 (a) OM and (b) AFM of spray-deposited P3HT on $\mathrm{Si} / \mathrm{SiO}_{x}$ from solution $1.5 \mathrm{~g} / \mathrm{L}$ in chloroform (120 s deposition time). (c) Absorption spectrum of P3HT in chloroform solution and in solid layer after spray deposition
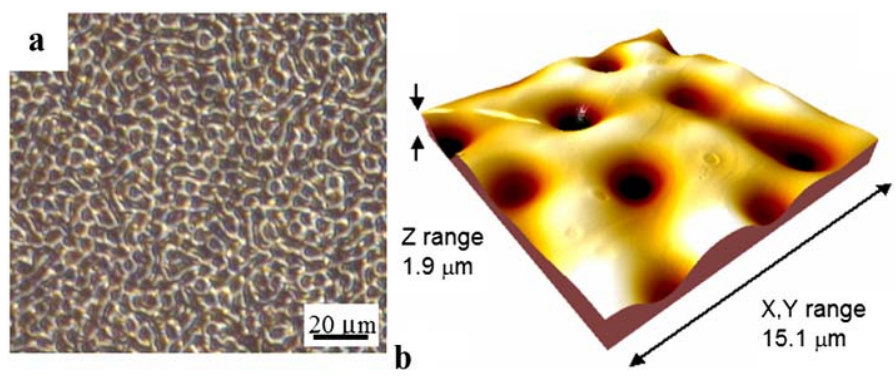

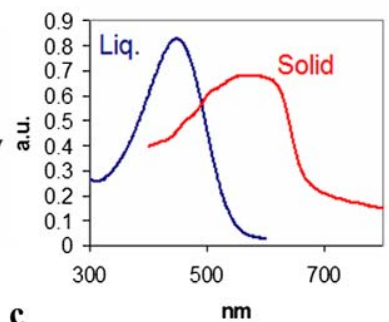

Figure 1 shows the optical microscopy (OM) images of P3HT deposited on a $\mathrm{Si} / \mathrm{SiO}_{x}$ substrate by spray deposition recorded at different times. On the millimetre scales the material deposition is uniform. On the micrometre scale a characteristic pattern is obtained, with short fibrils having a width of $3 \mu \mathrm{m}$, whose density rapidly increases, after $60 \mathrm{~s}$ leading to a complete coverage of the substrate. The morphology of these fibrils is different from the typical nanometric needles obtained by self-assembly of P3HT [22, 24, 40], and appears more similar to the morphology dictated by solution de-wetting [5, 41-43]. In particular, at all stages of the layer deposition it is possible to observe the presence of small holes in the organic layer, having a diameter of 1$5 \mu \mathrm{m}$. The density of these holes increases during deposition; at complete coverage (Fig. 2a) the holes form a continuous pattern on the surface. While a hole width ranges from 3 to $5 \mu \mathrm{m}$, its depth ranges from 0.4 up to $1.5 \mu \mathrm{m}$, leading to a P3HT morphology which is uniform on the macroscopic scale on all the prepared samples.

Atomic force microscopy (AFM) imaging (Fig. 2b) shows fine details of the deposited layer. The thickness of the sample is $\sim 3 \mu \mathrm{m}$. It is possible to distinguish two different relevant length scales to describe the surface morphology. While on tens of microns scale the $R_{\text {RMS }}$ amounts to $450 \pm 50 \mathrm{~nm}$, on the sub-micron scale this value decreases down to $0.5 \pm 0.2 \mathrm{~nm}$. This is due to the fact that on the large scale the $R_{\mathrm{RMS}}$ value is averaged over surface exposing both holes and flat regions. Despite these micrometric fluctuations, the surface appears to be very flat on the submicron scale. The $R_{\text {RMS }}$ on such a small scale has been calculated using the following procedure: (1) Each AFM image performed on a ten-micron scale have been sub-divided in portions having $1 \times 1 \mu^{2}$ size. (2) Each portion has been linearised by subtracting a 2D polynomial curve having a curvature larger than $1 \mu \mathrm{m}$. As reference, the $R_{\mathrm{RMS}}$ value of a clean $\mathrm{Si} / \mathrm{SiO}_{x}$ surface has also been measured and found to be $0.2 \mathrm{~nm}$ which can be primarily ascribed to the thermal noise effect [44].

Image analysis has been used to gain quantitative insight into the pattern uniformity. Figure 3 a shows a large scale OM image of the deposited layer in a film prepared with a $120 \mathrm{~s}$ deposition time. Fast Fourier transform (FFT) analysis (Fig. 3b) of these films reveals a hole periodicity of $5.0 \pm 0.7 \mu \mathrm{m}$. Autocorrelation analysis of the OM image (Fig. 3c) and the profile of the line traced (Fig. 3d) offers more details. The dark ring visible in Fig. $3 \mathrm{c}$ indicates that the minimal distance between holes is $5 \mu \mathrm{m}$, in good agreement with FFT results. Furthermore, the presence of ripples around the first ring indicates that hole correlation extends beyond next-nearest neighbours, up to ca. $10 \mu \mathrm{m}$. Both the dark ring and the ripples show a preferential hexagonal packing of holes which maximises their number. This evidence suggests that the density of impact points of the solvent drops on the substrate during the spray deposition may be assumed as uniform.

The presence of holes and rings upon spray deposition of polymers has been previously observed, and it is due to the collision of sprayed droplets with the surface [21]. Although random and uncorrelated, the continuous collision of solution with the substrate yields a very uniform morphology, with the holes adopting a close-packed arrangement. Though we could not measure in situ the size of solution droplets hitting the substrate, the hole size and spacing suggest that the spray deposition generates droplets of 3-5 $\mu \mathrm{m}$ in diameter, which splash on the surface depositing material and leaving holes due to the drop collision. The presence of 
Fig. 3 (a) Large scale OM of spray-deposited P3HT on $\mathrm{Si} / \mathrm{SiO}_{x}$ from solution $1.5 \mathrm{~g} / \mathrm{L}$ in chloroform (120 s deposition time), and (b) corresponding radially averaged FFT showing the $5 \mu \mathrm{m}$ peak. (c) Image of the autocorrelation function $S(x, y)$ displaying fluctuation rims in the hole-hole correlation density and its hexagonal symmetry, and (d) corresponding line profile $S(x, 0)$
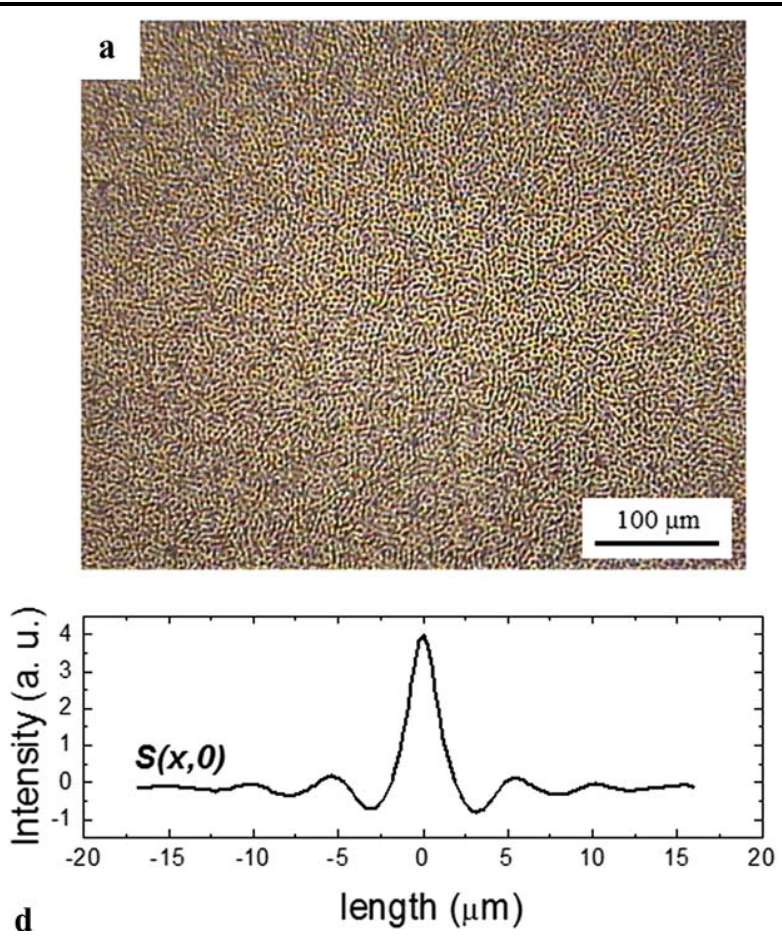
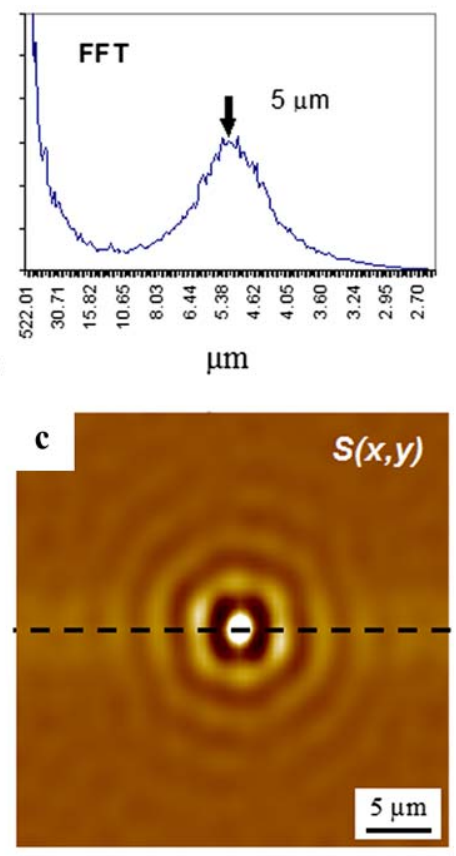

these holes at early and late stages of deposition, observed both on thin and thick layers, rules out the possibility that the holes are due to de-wetting on the $\mathrm{Si} / \mathrm{SiO}_{x}$ surface.

The peculiar micro-patterning obtained has also been observed on transparent glass substrates thus making it possible to perform optical absorption spectroscopy of the deposited layer, and to compare it with its absorption spectrum in solution. A strong red shift of absorption is observed (Fig. 2c) with absorption maxima passing from 450 to $\sim 580 \mathrm{~nm}$, due to the different inter-chromophore interactions. While in solution the thiophene units of the polymer backbone can twist, in the solid layer attractive inter-chain interactions result in the formation of packed structures in which the rotation about the interring bond is hindered extending the $\pi$ conjugation across multiple thiophenes and thus causing a decrease of optical band gap [45]. In other words, in solution, repulsive steric interactions between subsequent repeat units as well as the interaction of the "good" solvent with the alkyl side chains cause a twisting of the polymer backbone associated with a broad and feature-less absorption. In contrast, inter-chain interactions in the solid state result in the formation of a lamellar structure, in which the rotation about the interring bond is highly restricted. As a result, the polymer backbone in the solid state can be described as a flat, rigid-rod molecule, with more extended $\pi$ conjugation and red-shifted, solid-state adsorption [45].

We explored the possibility to use the peculiar morphology obtained for P3HT to template the growth of a second layer of material. We chose PDI as a test molecule, as its self-assembly behaviour has been extensively studied in pre- vious works [46-48], and because P3HT:PDI blends have been explored both on macroscopic [49] and microscopic [24] scales, and showed interesting photovoltaic properties.

When deposited on flat substrates, PDI molecules tend to aggregate in nano-sized, elongated crystals [46, 47]. Either spin coating or drop casting has been used to investigate the effect of the P3HT templating layer on this kind of self-assembly. PDI is soluble both in chloroform (which is a good solvent also for P3HT) or methanol (in which P3HT is insoluble). Upon spin coating of PDI from a chloroform solution, a partial re-dissolution of the deposited P3HT layer occurs, as proved by the partial destruction of the hole pattern, and the decrease in hole depth to less than $100 \mathrm{~nm}$ (not shown). In contrast, by using methanol as a solvent the P3HT morphology is perfectly preserved as confirmed through an experiment performed by spin coating methanol on the patterned P3HT surface revealing that the film morphology remains unaltered, thus excluding a re-dissolution of P3HT. A PDI film spin-coated from a $\mathrm{MeOH}$ solution on the templating P3HT film is shown in Figs. 4a, b. It displayed small crystals of PDI adsorbed preferentially into the P3HT holes. Due to the low amount of material deposited by spin coating, the crystals are observed only on some areas of the surface.

For the sake of comparison, Fig. $4 \mathrm{c}$ shows a PDI film obtained by spin coating a solution in methanol on a bare $\mathrm{Si} / \mathrm{SiO}_{x}$ surface. It reveals self-assembled elongated nanocrystals with dimensions and shapes similar to those already observed using other solvents [46, 47]. The PDI crystals grown on the P3HT templating layer (Figs. 4a, b) appear 


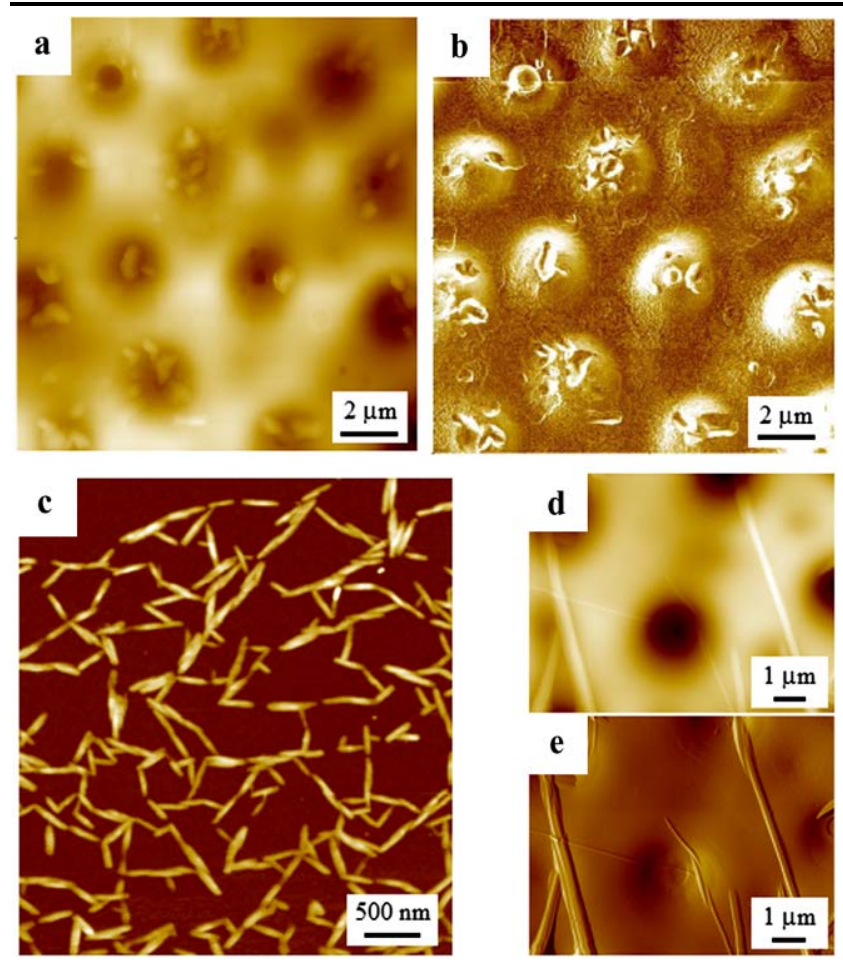

Fig. 4 (a) AFM topography and (b) phase image of nano-crystals grown onto P3HT by spin coating from a saturated solution of PDI in $\mathrm{MeOH}$. (c) AFM of the same nano-crystals grown onto neat $\mathrm{Si} / \mathrm{SiO}_{x}$ by spin coating a saturated solution of PDI in $\mathrm{MeOH}$. (d) AFM topography and (e) corresponding $\mathrm{X}$-gradient image of nano-crystals grown onto P3HT by drop casting from a saturated solution of PDI in $\mathrm{MeOH}$. Z-range (a) $1.7 \mu \mathrm{m}$, (b) $51 \mathrm{deg}$, (c) $22 \mathrm{~nm}$, (d) $1,7 \mu \mathrm{m}$ and (e) $4.7 \mu \mathrm{m} / \mu \mathrm{m}$

slightly less elongated due to the different chemical nature of the substrate [5].

As the underlying layer is composed of P3HT, preferential growth cannot be ascribed to differences in surface chemical functionalisation, but rather to the propensity of the solution to evaporate first from the most convex areas of the surface, forming droplets in correspondence to the holes in order to minimize surface tension. The final result is a surface with microscopic holes of P3HT, with one or more PDI crystals present in some of the holes. In this kind of morphology the P3HT:PDI phase separation is on the micrometre scale, thus very different from P3HT:PDI blends used for photovoltaic applications, where phase separation is required on the nano-metric scale. In contrast, by depositing a greater amount of PDI on the P3HT patterned surface by drop casting, larger crystals with a fibre-like morphology are formed as a result of the slow solvent evaporation and self-assembly process $[46,50]$. Differently, these fibres have been found to be randomly located on the patterned surface (Figs. 4d, e).

\section{Conclusions}

In summary, we have exploited a two-step deposition method from solution using sub-sequential spray deposition of P3HT and spin coating or drop casting of an alkyl substituted PDI in orthogonal solvents. We have shown that macroscopic periodic patterns of P3HT can be formed and exploited to template the growth of PDI crystals in particular areas of the surface. This combined approach of spray deposition and spin coating or drop casting is easily upscalable and highly versatile as it can be applied to various materials leading to the generation of new complex and multi-component un-conventional morphologies, which are not attainable with other more classical techniques.

\section{References}

1. A.C. Grimsdale, K. Müllen, Angew. Chem. Int. Ed. 44, 5592 (2005)

2. J.P. Rabe, S. Buchholz, Science 253, 424 (1991)

3. H.J. Räder, A. Rouhanipour, A.M. Talarico, V. Palermo, P. Samorì, K. Müllen, Nat. Mater. 5, 276 (2006)

4. P. Leclère, M. Surin, P. Brocorens, M. Cavallini, F. Biscarini, R. Lazzaroni, Mater. Sci. Eng. Rep. 55, 1 (2006)

5. V. Palermo, P. Samorì, Angew. Chem. Int. Ed. 46, 4428 (2007)

6. P.G. De Gennes, Rev. Mod. Phys. 57, 827 (1985)

7. F. Cacialli, J.S. Wilson, J.J. Michels, C. Daniel, C. Silva, R.H. Friend, N. Severin, P. Samorì, J.P. Rabe, M.J. O’Connell, P.N. Taylor, H.L. Anderson, Nat. Mater. 1, 160 (2002)

8. S.R. Forrest, Nature 428, 911 (2004)

9. R.H. Friend, R.W. Gymer, A.B. Holmes, J.H. Burroughes, R.N. Marks, C. Taliani, D.D.C. Bradley, D.A. Dos Santos, J.L. Brédas, M. Logdlund, W.R. Salaneck, Nature 397, 121 (1999)

10. K. Müllen, J.P. Rabe, Acc. Chem. Res. 41, 511 (2008)

11. R. van Hameren, P. Schon, A.M. van Buul, J. Hoogboom, S.V. Lazarenko, J.W. Gerritsen, H. Engelkamp, P.C.M. Christianen, H.A. Heus, J.C. Maan, T. Rasing, S. Speller, A.E. Rowan, J. Elemans, R.J.M. Nolte, Science 314, 1433 (2006)

12. D.J. Vak, S.S. Kim, J. Jo, S.H. Oh, S.I. Na, J.W. Kim, D.Y. Kim, Appl. Phys. Lett. 91 (2007)

13. R. Green, A. Morfa, A.J. Ferguson, N. Kopidakis, G. Rumbles, S.E. Shaheen, Appl. Phys. Lett. 92 (2008)

14. K. Fujita, T. Ishikawa, T. Tsutsui, Jpn. J. Appl. Phys., Part 241 , L70 (2002)

15. Y. Okabayashi, T. Mitarai, S. Yamazaki, R. Matsuyama, K. Kanai, Y. Ouchi, K. Seki, Appl. Surf. Sci. 244, 217 (2005)

16. T. Ishikawa, M. Nakamura, K. Fujita, T. Tsutsui, Appl. Phys. Lett. 84, 2424 (2004)

17. T. Ishikawa, K. Fujita, T. Tsutsui, Jpn. J. Appl. Phys., Part 144 , 6292 (2005)

18. J.U. Park, M. Hardy, S.J. Kang, K. Barton, K. Adair, D.K. Mukhopadhyay, C.Y. Lee, M.S. Strano, A.G. Alleyne, J.G. Georgiadis, P.M. Ferreira, J.A. Rogers, Nat. Mater. 6, 782 (2007)

19. X.L. Mo, T. Mizokuro, H. Mochizuki, N. Tanigaki, T. Hiraga, Jpn. J. Appl. Phys., Part 1 44, 656 (2005)

20. A. Izquierdo, S.S. Ono, J.C. Voegel, P. Schaaff, G. Decher, Langmuir 21, 7558 (2005)

21. X. Mo, T. Mizokuro, N. Tanigaki, T. Hiraga, N. Umehara, K. Takagi, S. Yamamoto, Jpn. J. Appl. Phys. 47, 425 (2008)

22. J.A. Merlo, C.D. Frisbie, J. Phys. Chem. B 108, 19169 (2004) 
23. J.Y. Kim, K. Lee, N.E. Coates, D. Moses, T.Q. Nguyen, M. Dante, A.J. Heeger, Science 317, 222 (2007)

24. A. Liscio, G. De Luca, F. Nolde, V. Palermo, P. Samorì, K. Müllen, J. Am. Chem. Soc. 130, 780 (2008)

25. J.A.A.W. Elemans, R. Van Hameren, R.J.M. Nolte, A.E. Rowan, Adv. Mater. 18, 1251 (2006)

26. F. Würthner, Chem. Commun. 1564 (2004)

27. K. Balakrishnan, A. Datar, T. Naddo, J. Huang, R. Oitker, M. Yen, J. Zhao, L. Zang, J. Am. Chem. Soc. 128, 7390 (2006)

28. K. Balakrishnan, A. Datar, R. Oitker, H. Chen, J.M. Zuo, L. Zang, J. Am. Chem. Soc. 127, 10496 (2005)

29. F. Dinelli, R. Capelli, M.A. Loi, M. Murgia, M. Muccini, A. Facchetti, T.J. Marks, Adv. Mater. 18, 1416 (2006)

30. X. Zhang, Z. Chen, F. Würthner, J. Am. Chem. Soc. 129, 4886 (2007)

31. F. Nolde, J.Q. Qu, C. Kohl, N.G. Pschirer, E. Reuther, K. Müllen, Chem. Eur. J. 11, 3959 (2005)

32. W. Kern, D.A. Puotinen, RCA Rev. Tech. J. 31, 187 (1970)

33. P. Samorì, Chem. Soc. Rev. 34, 551 (2005)

34. S.S. Sheiko, M. Möller, Chem. Rev. 101, 4099 (2001)

35. H. Takano, J.R. Kenseth, S.-S. Wong, J.C. O'Brien, M.D. Porter, Chem. Rev. 99, 2845 (1999)

36. M.O. Finot, M.T. McDermott, J. Am. Chem. Soc. 119, 8564 (1997)

37. P. Leclère, R. Lazzaroni, J.L. Brédas, J.M. Yu, P. Dubois, R. Jérôme, Langmuir 12, 4317 (1996)

38. P. Samorì, M. Surin, V. Palermo, R. Lazzaroni, P. Leclère, Phys. Chem. Chem. Phys. 8, 3927 (2006)
39. Scanning Probe Image Processor (SPIP) (version 2.000, Image Metrology ApS)

40. H.C. Yang, T.J. Shin, L. Yang, K. Cho, C.Y. Ryu, Z.N. Bao, Adv. Funct. Mater. 15, 671 (2005)

41. E. Rabani, D.R. Reichman, P.L. Geissler, L.E. Brus, Nature 426, $271(2003)$

42. R.D. Deegan, O. Bakajin, T.F. Dupont, G. Huber, S.R. Nagel, T.A. Witten, Nature 389, 827 (1997)

43. S. Herminghaus, K. Jacobs, K. Mecke, J. Bischof, A. Fery, M. IbnElhaj, S. Schlagowski, Science 282, 916 (1998)

44. T.E. Schäffer, Nanotechnology 16, 664 (2005)

45. A. Zen, J. Pflaum, S. Hirschmann, W. Zhuang, F. Jaiser, U. Asawapirom, J.P. Rabe, U. Scherf, D. Neher, Adv. Funct. Mater. 14, 757 (2004)

46. V. Palermo, A. Liscio, D. Gentilini, F. Nolde, K. Müllen, P. Samorì, Small 3, 161 (2006)

47. G. De Luca, A. Liscio, P. Maccagnani, F. Nolde, V. Palermo, K. Müllen, P. Samorì, Adv. Funct. Mater. 17, 3791 (2007)

48. A.L. Briseno, S.C.B. Mannsfeld, C. Reese, J.M. Hancock, Y. Xiong, S.A. Jenekhe, Z. Bao, Y. Xia, Nano Lett. 7, 2847 (2007)

49. W.S. Shin, H.H. Jeong, M.K. Kim, S.H. Jin, M.R. Kim, J.K. Lee, J.W. Lee, Y.S. Gal, J. Mater. Chem. 16, 384 (2006)

50. P. Samorì, M. Keil, R. Friedlein, J. Birgerson, M. Watson, M. Müllen, W.R. Salaneck, J.P. Rabe, J. Phys. Chem. B 105, 11114 (2001) 